\title{
Role of nitric oxide and prostaglandin in the maintenance of cortical and renal medullary blood flow
}

\author{
S.I. Gomez, D.M. Strick and J.C. Romero \\ Department of Physiology and Biomedical Engineering, Mayo Clinic College of Medicine, Rochester, \\ MN, USA \\ Correspondence to: J.C. Romero, Mayo Clinic, Stabile 7, 200 First Street SW, Rochester, MN 55905, USA \\ Fax: +1-507-266-9316. E-mail: romero.juan@mayo.edu
}

\begin{abstract}
This study was undertaken in anesthetized dogs to evaluate the relative participation of prostaglandins (PGs) and nitric oxide (NO) in the maintenance of total renal blood flow (TRBF), and renal medullary blood flow (RMBF). It was hypothesized that the inhibition of NO should impair cortical and medullary circulation because of the synthesis of this compound in the endothelial cells of these two territories. In contrast, under normal conditions of perfusion pressure PG synthesis is confined to the renal medulla. Hence PG inhibition should predominantly impair the medullary circulation. The initial administration of $25 \mu \mathrm{M} \mathrm{kg}{ }^{-1} \mathrm{~min}^{-1}$ NG-nitro-L-arginine methyl ester produced a significant $26 \%$ decrease in TRBF and a concomitant $34 \%$ fall in RMBF, while the subsequent inhibition of PGs with $5 \mathrm{mg} / \mathrm{kg}$ meclofenamate further reduced TRBF by $33 \%$ and RMBF by $89 \%$. In contrast, the initial administration of meclofenamate failed to change TRBF, while decreasing RMBF by $49 \%$. The subsequent blockade of NO decreased TRBF by $35 \%$ without further altering RMBF. These results indicate that initial PG synthesis inhibition predominantly alters the medullary circulation, whereas NO inhibition decreases both cortical and medullary flow. This latter change induced by NO renders cortical and RMBF susceptible to a further decrease by PG inhibition. However, the decrease in medullary circulation produced by NO inhibition is not further enhanced by subsequent PG inhibition.
\end{abstract}

Key words: Kidney; NG-nitro-L-arginine methyl ester; Meclofenamate; Renal circulation

Research supported by National Institutes of Health, grant HL16496, and by the Mayo Foundation.

Received April 2, 2007. Accepted October 30, 2007

\section{Introduction}

The relative role of naturally occurring vasodilators in the renal circulation has remained largely ill defined. However, identification of their mechanism of action is of critical importance to understand how the kidney influences the equilibrium of body fluids and blood pressure (1-4). Of particular interest are the effects of prostaglandins (PGs) and nitric oxide (NO), both of which exhibit a high rate of synthesis in the renal medulla (5-7) and in the renal vascular endothelium (8). In fact there are observations showing that the blockade of NO produces, in the rat, a decrease in medullary circulation which leads with time to excessive sodium retention and a sustained increase in blood pres- sure $(9,10)$. Similarly, an increase in diastolic blood pressure has been observed in humans after prolonged blockade of PG synthesis with indomethacin (11). In spite of this, no study has been conducted to determine the specific changes occurring in cortical and medullary blood flow when NO and PGs (or vice versa) are inhibited in succession. This approach implies the definition not only of the initial effect produced by the inhibition of the synthesis of each of these substances, but also the determination of how the inhibition of one substance affects the response to the other.

The present study was undertaken to answer these questions. Total renal blood flow (TRBF) was measured with an electromagnetic flow meter (12) while renal medul- 
lary blood flow (RMBF) was continuously measured using a laser Doppler flow probe (12). Two basic experimental protocols were used. In the first, flow determinations were made under basal control conditions, as well as after the blockade of NO and PG synthesis with NG-nitro-L-arginine methyl ester (L-NAME) and meclofenamate (MECLO), respectively. In the second, the order of $P G$ and NO inhibition was reversed. The relative effectiveness of LNAME and MECLO in blocking the production of NO and PGs was evaluated by measuring the vasodilator response to bradykinin (BK).

\section{Material and Methods}

Experiments were performed on 14 male mongrel dogs (body weight $16-23 \mathrm{~kg}$ ) maintained on a standard laboratory diet with free access to water. The animals were fasted the evening before the surgery. On the morning of the experiments, the dogs were anesthetized with sodium pentobarbital $(30 \mathrm{mg} / \mathrm{kg}$ ) and ventilated mechanically with a Harvard respirator (South Natick, MA, USA) at a tidal volume appropriate for body weight based on the nomogram of Kleinman and Radford Jr. (13). A catheter was inserted into a femoral artery and advanced into the aorta to a position just below the renal arteries for measurement of renal perfusion pressure (RPP) using a Statham P23ID pressure transducer (Viggo-Spectramed, Oxnard, CA, USA), and for collection of peripheral arterial blood samples. A femoral vein was cannulated for continuous infusions of $0.9 \%$ saline. The volume of saline infused into the animals by the time the experiments were started $(\sim 60 \mathrm{~mL})$ was sufficient to replace any blood lost during surgery and to render the animals euvolemic. In addition, sodium pentobarbital $\left(\sim 4 \mathrm{mg} \mathrm{kg}^{-1} \mathrm{~h}^{-1}\right)$ was infused to maintain a constant plane of anesthesia throughout the experiments.

The left kidney was exposed through a flank incision and the dogs were then suspended in a metal frame that mimicked their usual standing position. A non-cannulating electromagnetic flow probe (Carolina Medical Electronics, King, NC, USA) of suitable diameter was placed on the renal artery at its origin from the aorta for the measurement of total renal blood flow. A zero flow baseline signal for the probe was established at the beginning of each experiment and was checked at the conclusion of each protocol by occluding the renal artery distal to the probe with a snare made of a silk ligature enclosed in polyethylene tubing. A curved 23-gauge needle attached to polyethylene tubing and connected to a syringe pump (Harvard model 944) was inserted into the renal artery for continuous infusion of $0.9 \%$ saline, BK, or L-NAME at a rate of 1 $\mathrm{mL} / \mathrm{min}$. The kidney was then further prepared for meas- urement of RMBF by creating a cortical window in an alcohol-injected region of the kidney using an $\mathrm{He}-\mathrm{Ne}-\mathrm{CO}_{2}$ beam surgical laser and placing a multiple optical fiber flow probe (model PF 315:72; Perimed, Inc., Stockholm, Sweden) connected to a Perimed PF laser Doppler flowmeter directly on the medulla near the renal crest. This procedure has been published in detail elsewhere (12). Zero flow baseline laser Doppler signals were determined at the beginning of each experiment by occluding the renal artery for several seconds. After completion of the surgical procedure, a 1-h stabilization period was allowed to elapse before starting the experiments. The Doppler flowmeter signal was conventionally normalized to $100 \%$ for every experiment.

\section{Protocol 1}

Baseline RPP, TRBF, and RMBF values were recorded. The intrarenal infusion of saline was then replaced with an infusion of BK (4 pM kg-1 $\mathrm{min}^{-1} ; 1 \mathrm{~mL} / \mathrm{min}$ ) which was continued for $10 \mathrm{~min}$. Results of preliminary studies from our laboratory have shown that the administration of BK in this manner consistently produced peak increases in TRBF and associated increases in RMBF within 1-2 min after the beginning of infusion. Both parameters then declined toward a steady-state level over the remainder of the infusion. The responses to BK were recorded during the peak (1-2 $\mathrm{min}$ ) and during the steady-state condition (10 $\mathrm{min}$ ). After $10 \mathrm{~min}$, BK infusion was stopped and a 30-min recovery period was allowed. Following the recovery period, an intrarenal infusion of L-NAME $\left(25 \mu \mathrm{M} \mathrm{kg}^{-1} \mathrm{~min}^{-1}\right)$, an inhibitor of $\mathrm{NO}$ synthesis, was started and 20 min were allowed for renal parameters to stabilize. The administration of L-NAME was continued and a simultaneous intrarenal infusion of BK was started. Both infusions were continued for $10 \mathrm{~min}$ before being stopped and replaced with saline. Another 30 -min recovery period was allowed before an intravenous bolus injection of $5 \mathrm{mg} / \mathrm{kg} \mathrm{MECLO}$, an inhibitor of PG synthesis, was administered. Forty minutes were allowed for the intrarenal effects of the drug to become manifest before a final intrarenal BK infusion was begun. RPP, TRBF, and RMBF were recorded immediately before, and 20 and $40 \mathrm{~min}$ after the administration of L-NAME and MECLO, respectively. The latter values were recorded immediately before the BK infusions and thus were also the baseline values for the subsequent $\mathrm{BK}$ responses. The peak and 10-min responses of these parameters to $\mathrm{BK}$ infusion were also recorded.

\section{Protocol 2}

This protocol was identical to the first, except that the order of L-NAME and MECLO administration was reversed. 
BK was initially infused intrarenally for 10 min to determine TRBF and RMBF responses. Thirty minutes after the end of the BK infusion, an intravenous bolus of MECLO (5 mg/ $\mathrm{kg}$ ) was administered and 40 min were allowed for parameters to stabilize. Another identical BK infusion was administered a second time for $10 \mathrm{~min}$. After the end of $\mathrm{BK}$ infusion, a 30-min recovery period was allowed before an intrarenal infusion of L-NAME was started. Twenty minutes after initiating the administration of L-NAME, a simultaneous infusion of BK was started and both infusions were continued for an additional $10 \mathrm{~min}$. RPP, TRBF, and RMBF were recorded as described above.

At the end of each experiment, the left kidney was removed, sectioned along the lines of tissue infarction caused by alcohol injection, and weighed to determine the weights of perfused and non-perfused tissue (12). TRBF was then normalized per gram of perfused kidney weight.

\section{Statistical analyses}

Data are reported as means \pm SEM. RPP, TRBF, and $\mathrm{RMBF}$ data were compared by repeated-measures analysis of variance, followed by Newman-Keuls tests when a significant $F$ value was calculated $(14,15)$. All statistical analyses were considered to be significant if $P<0.05$.

\section{Results}

It should first be observed that in Figures 1 and 2 the baseline values of RPP, TRBF, and RMBF were conventionally adjusted to $100 \%$. This allows one to depict the alterations induced by any of the inhibitors of PGs or NO or by the rapid infusion of $\mathrm{BK}$ as the percent change of control values.

It can be seen in Figure 1 that the control levels of TRBF were significantly increased $(P<0.05)$ by the intrarenal infusion of BK from $3.6 \pm 0.5$ to $4.7 \pm 0.7 \mathrm{~mL} \mathrm{~g}^{-1}$ $\mathrm{min}^{-1}$. BK failed to produce any significant change in either RMBF (from $411.4 \pm 69$ to $431.4 \pm 80$ conventional units) or in RPP (from $128 \pm 3.5$ to $124 \pm 3.2 \mathrm{mmHg}$ ). Thirty minutes later, the inhibition of NO with L-NAME resulted in a significant fall in TRBF from $3.28 \pm 0.4$ to $2.40 \pm 0.3 \mathrm{~mL} \mathrm{~g}^{-1} \mathrm{~min}^{-1}$. Similarly, L-NAME induced a significant decrease in RMBF from $327 \pm 68$ to $215 \pm 46$ conventional units. However, LNAME produced a mild but significant increase $(P<0.05)$ in RPP from $126 \pm 5$ to $133 \pm 4 \mathrm{mmHg}$. None of these changes produced by L-NAME in TRBF or RMBF did affect the vasodilator response to $\mathrm{BK}$, which significantly increased TRBF $(P<0.05)$ from $2.4 \pm 0.3$ to $3.4 \pm 5 \mathrm{~mL} \mathrm{~g}^{-1}$ $\mathrm{min}^{-1}$. Similarly, RMBF increased from $215 \pm 46$ to $351 \pm 65$ $\mathrm{mL} \mathrm{g}^{-1} \mathrm{~min}^{-1}$. These changes in TRBF and RMBF represent an increase of 41 and $63 \%$, respectively, over preinfusion values. Under these conditions, the inhibition of PGs by the intrarenal infusion of MECLO produced a further decrease in both TRBF (from $2.3 \pm 0.3$ to $1.56 \pm 3 \mathrm{~mL} \mathrm{~g}^{-1} \mathrm{~min}^{-1}$ ) and RMBF (from $185 \pm 42$ to $20 \pm 6$ conventional units). Furthermore, Figure 1 shows that, after MECLO, the administration of $\mathrm{BK}$ failed to induce any significant increase in either TRBF or RMBF.

Figure 2 shows the same parameters as those illustrated in Figure 1, except that the order of the inhibitors administered was reversed (i.e., MECLO was given before L-NAME). Doses and routes of administration were the same used in Figure 1. Infusion of BK alone in this group of dogs significantly increased $(P<0.001)$ RMBF from 211.1 \pm 27 to $261 \pm 39$ conventional units and TRBF from $3.6 \pm$ 0.6 to $5.6 \pm 7.1$ above baseline. However, BK failed to alter RPP (from $126 \pm 3$ to $125 \pm 3 \mathrm{mmHg}$ ). Administration of MECLO had no significant effect on TRBF (from $4.3 \pm 1$ to $4.6 \pm 0.5 \mathrm{~mL} \mathrm{~g}^{-1} \mathrm{~min}^{-1}$ ) but significantly decreased RMBF from $169 \pm 26$ to $81 \pm 26$ conventional units. Intrarenal infusion of BK during inhibition of PG synthesis did not alter significantly TRBF but it did change RMBF from $81 \pm 26$ to $201 \pm 34$ conventional units $(P<0.01)$. Inhibition of intrarenal

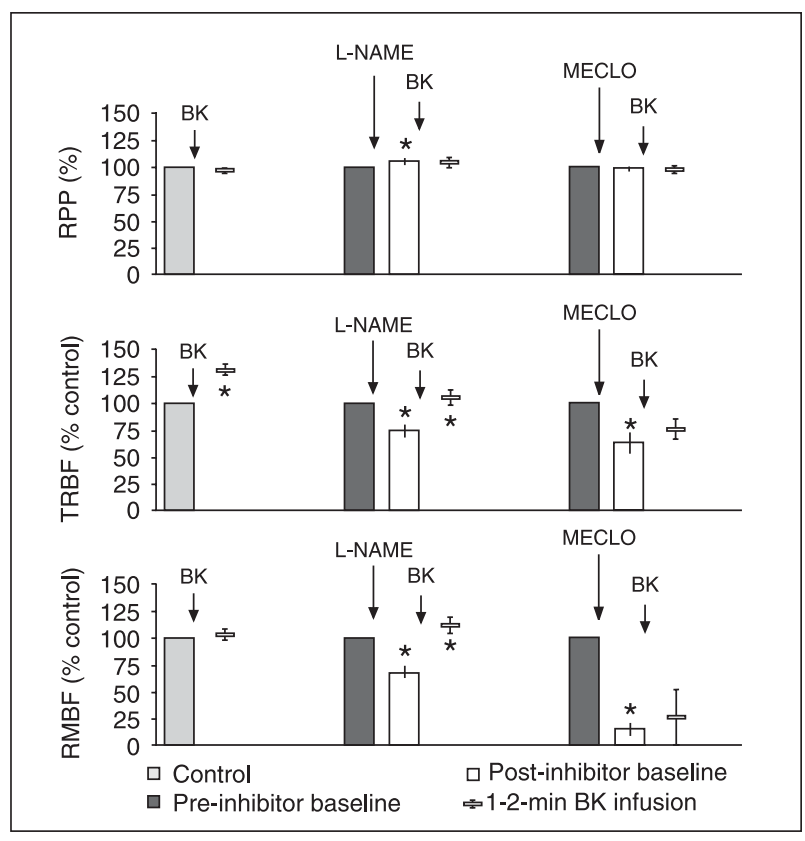

Figure 1. The response of renal perfusion pressure (RPP), total renal blood flow (TRBF), and renal medullary blood flow (RMBF) to intrarenal infusion of bradykinin (BK; $4 \mathrm{pM} \mathrm{kg}^{-1} \mathrm{~min}^{-1} ; 1 \mathrm{~mL} /$ min) followed by the sequential intrarenal infusion of $25 \mu \mathrm{M} \mathrm{kg}^{-1}$ $\mathrm{min}^{-1}$ NG-nitro-L-arginine methyl ester (L-NAME) and then an intravenous bolus injection of meclofenamate (MECLO; $5 \mathrm{mg} /$ kg). ${ }^{*} \mathrm{P}<0.05$ compared to pre-inhibitor baseline recorded immediately before the injection (ANOVA). 
NO synthesis by L-NAME during continued PG synthesis blockade had no further effect on RMBF $(69.3 \pm 26$ to $35 \pm$ 10 conventional units) but decreased TRBF significantly by $35 \%\left(4.5 \pm 0.6\right.$ to $\left.2.9 \pm 0.8 \mathrm{~mL} \mathrm{~g}^{-1} \mathrm{~min}^{-1}\right)$. BK infusion after L-NAME administration did not significantly alter TRBF (3.7 \pm 0.8 to $\left.3.2 \pm 0.7 \mathrm{~mL} \mathrm{~g}^{-1} \mathrm{~min}^{-1}\right)$ or RMBF $(69 \pm 26$ to 35.5 \pm 10 conventional units). RPP was increased slightly by the administration of L-NAME after MECLO (126 \pm 4 to 141 $\pm 5 \mathrm{mmHg}$ ). Plasma hematocrit averaged $50 \pm 2 \%$. Total kidney weight averaged $31 \pm 2 \mathrm{~g}$ and the average weight of the perfused kidney sections after alcohol infusion was 18 $\pm 1 \mathrm{~g}$.

\section{Discussion}

There is, at present, a well accepted notion that PGs $(9,10)$ and $N O(5,16,17)$ play an important role in the regulation of TRBF. In fact, previous studies have shown that the blockade of either NO or PG is followed by a decrease in TRBF and impairment in urine sodium excretion both under basal conditions $(5,6)$ and during pressureinduced natriuresis $(6,7,18)$. However, the specific effect of the inhibition of each of these substances when com-

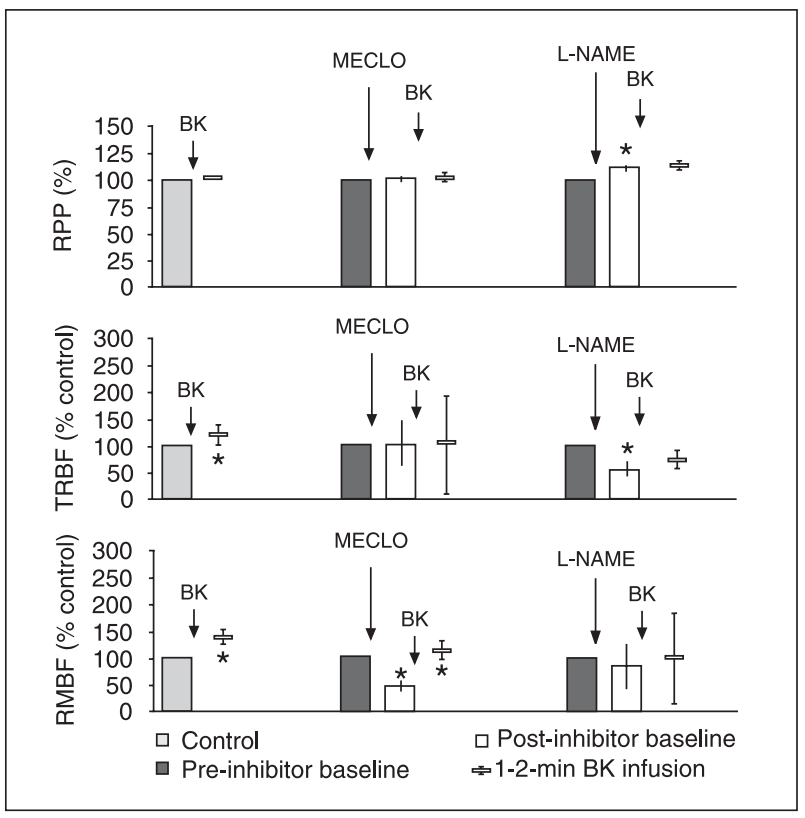

Figure 2. The response of renal perfusion pressure (RPP), total renal blood flow (TRBF), and renal medullary blood flow (RMBF) to intrarenal infusion of bradykinin (BK; $4 \mathrm{pM} \mathrm{kg}^{-1} \mathrm{~min}^{-1} ; 1 \mathrm{~mL} /$ min) followed by the sequential intravenous bolus injection of meclofenamate (MECLO; $5 \mathrm{mg} / \mathrm{kg}$ ) and then intrarenal infusion of $25 \mu \mathrm{M} \mathrm{kg}^{-1} \mathrm{~min}^{-1} \mathrm{NG}$-nitro-L-arginine methyl ester (L-NAME). ${ }^{*} \mathrm{P}<0.05$ compared to pre-inhibitor baseline recorded immediately before the injection (ANOVA). pared under similar conditions or under conditions where one of them had been previously abolished has never been tested. This prompted us to perform the present study in which we assessed the relative significance of $\mathrm{NO}$ and $P G$ in the maintenance of both RMBF and TRBF under resting conditions and after the consecutive inhibition of $\mathrm{PGs}$ and/or NO. The results confirmed our assumption that the inhibition of NO synthesis with the administration of LNAME produced a significant decrease of both TRBF by $26 \%$, and RMBF by $34 \%$. In contrast, the initial inhibition of PG synthesis with indomethacin induced a significant $49 \%$ fall in RMBF without altering TRBF. It was also observed that the initial effects of each of these inhibitors were altered when given after the response of the other had been produced. The results also showed that the initial effect of each of these inhibitors, L-NAME or MECLO, was altered when one inhibitor was administered after the response to the other had been obtained.

To a large extent, the primary and secondary effects of $P G$ and NO inhibition can be understood on the basis of previous experimental findings showing that the efficacy of renal vasodilators in modulating renal, and particularly medullary, circulation is mainly determined by the level of $\operatorname{RPP}(5,19,20)$. Fenoy et al. (21), in agreement with our observation, observed that the administration of L-NAME to rats failed to produce significant changes in RMBF in kidneys perfused at normal levels of RPP. However, the inhibition of NO synthesis in kidneys perfused at higher RPP (140 mmHg) was followed by significant decreases in medullary blood flow. The authors interpreted these results as indicating that renal NO synthesis at high levels of perfusion pressure is largely responsible for maintaining medullary vasodilatation. Since in anesthetized normal dogs basal blood pressure $(125 \pm 5 \mathrm{mmHg})$ is slightly elevated when compared to non-anesthetized normotensive dogs $(110 \pm 5)$, we thought that this might predispose the kidney to be more responsive to inhibition of $\mathrm{NO}$ synthesis. In fact, the initial NO synthesis inhibition at a perfusion pressure of $125 \pm 5 \mathrm{mmHg}$ was followed by a significant decrease in TRBF as well as RMBF. In contrast to the effects observed after the blockade of NO synthesis, the inhibition of PG synthesis is followed by a marked reduction of renal blood flow when perfused at low, but not at high RPP levels $(5,7,16,19)$. This is in agreement with our results showing that PG inhibition did not alter TRBF. However, it produced a significant decrease of RMBF even under normal perfusion pressure. This suggests that $P G$ synthesis is important to maintain RMBF under normal conditions. It should be emphasized that the specific effect of PG inhibition at different levels of pressure on RMBF has not been studied, although it has been well established 
that PGs have a protective effect on the renal circulation during a decrease in perfusion pressure $(5,7,11)$.

In the present study, the efficacy of L-NAME and MECLO in inhibiting NO and PGs was assessed by the decrease caused by this inhibitor in the endothelium-dependent vasodilator effects of BK. In this respect, BK was used according to the characteristics shown by previous studies, i.e., that the simultaneous stimulation of $P G$ and NO synthesis produced by any endothelium-dependent vasodilator is not altered when only one of these substances (i.e., NO or PG) is inhibited (22). In the present study, we confirmed this characteristic, since the vasodilator response to BK was increased after the blockade of either PG or NO, but these responses were abolished after both NO and PG synthesis was inhibited. Mean arterial pressure (MAP) was not altered by any of the surgical procedures performed on the kidney or by the intrarenal infusion of BK. This latter observation indicates that the actions of BK were mainly limited to the renal vasculature.

The reason why the blockade of one vasodilator compensates for the actions of the other is not known, nor is it known why there are circumstances in which the inhibition of NO or PGs enhances the vasodilator effect of the other.

The findings of this study conducted on anesthetized dogs with the systematic inhibition of PGs or NO can be summarized as follows: 1) NO inhibition produced a systematic decrease of cortical circulation when performed before or after PG inhibition; 2) conversely, PG inhibition produced a systematic decrease in medullary circulation when performed before or after NO inhibition; 3) NO inhibition decreased medullary circulation only when NO was given before MECLO, and 4) PG inhibition affected cortical blood flow only when given after NO inhibition. All of these observations indicate that $\mathrm{NO}$ has a consistent influence on the cortical circulation, whereas PG exhibits a predominant effect on the medullary circulation. Conversely, it would appear that the effects of PG on the cortical circulation and of $\mathrm{NO}$ on the medullary circulation became noticeable after the other vasodilator had been inhibited.

It should be mentioned that preservation of renal medullary circulation appears to be important, not only to

\section{References}

1. Stein JH, Ferris TF, Huprich JE, Smith TC, Osgood RW. Effect of renal vasodilatation on the distribution of cortical blood flow in the kidney of the dog. J Clin Invest 1971; 50: 1429-1438.

2. Hardaker WT Jr, Wechsler AS. Redistribution of renal intracortical blood flow during dopamine infusion in dogs. Circ Res 1973; 33: 437-444. maintain the ability of the kidney to concentrate urine, but also to preserve sodium balance and thereby to maintain blood pressure $(9,10,22)$. Furthermore, in some animal species such as the rat, significant elevations of systemic blood pressure have been obtained after selective NO inhibition in the renal medulla (10). These effects are not as evident when PG synthesis is inhibited. Our study does not allow us to draw further conclusions on these issues because a) the changes induced by PG or NO synthesis inhibitors were acutely limited to a short period of time (40 $\mathrm{min}$ ), and $b$ ) the homeostasis of the renal medullary circulation in the dog may differ significantly from that of the rat (18). In the rat, changes in RPP above $75-80 \mathrm{mmHg}$ are accompanied by good cortical autoregulation, while autoregulatory responses in the medulla are conspicuously absent (23). However, in the dog, Majid et al. (18) observed that the medullary circulation exhibits an autoregulatory response that does not differ from that observed in the cortical vasculature. Similarly, Strick et al. (12) reported a similar finding in the dog, except that the autoregulatory response of the renal medulla was not as efficient as in the cortex in the lower range of autoregulation. Furthermore, investigations on intrarenal blood flow redistribution with electron beam computerized tomography have shown in the dog that increments of RPP within the range of autoregulation are accompanied by some redistribution of blood perfusion between the outer and inner medullary zone without altering overall medullary flow (24).

The significance of PGs in the maintenance of the medullary circulation should be further explored. In normotensive humans, chronic inhibition of PGs leads to decrements in plasma renin activity, hypoaldosteronism, and a sustained increase in diastolic pressure (11). There is also evidence showing that, in the rat, inhibition of $\mathrm{NO}$ produces a sustained decrease in medullary blood flow and hypertension. Bergström and Evans (25) have suggested that differences in medullary function and their importance for the maintenance of blood pressure may vary widely in different animal species.
3. Riley RJ. Effects of acute changes in renal cortical blood flow distribution on renal function in dogs. In: Wesson CG, Finelli GM Jr (Editors), Recent advances in renal physiology and pharmacology. Baltimore: University Park Press; 1974. p 149-163.

4. Zou AP, Cowley AW Jr. Nitric oxide in renal cortex and medulla. An in vivo microdialysis study. Hypertension 1997; 
29: $194-198$

5. Dunn MJ, Zambraski EJ. Renal effects of drugs that inhibit prostaglandin synthesis. Kidney Int 1980; 18: 609-622.

6. Romero JC, Knox FG. Mechanisms underlying pressurerelated natriuresis: the role of the renin-angiotensin and prostaglandin systems. State of the art lecture. Hypertension 1988; 11: 724-738.

7. Romero JC, Bentley MD, Vanhoutte PM, Knox FG. Intrarenal mechanisms that regulate sodium excretion in relationship to changes in blood pressure. Mayo Clin Proc 1989; 64: 1406-1424.

8. Romero JC, Lahera V, Salom MG, Biondi ML. Role of the endothelium-dependent relaxing factor nitric oxide on renal function. J Am Soc Nephrol 1992; 2: 1371-1387.

9. Cowley AW, Roman RJ, Fenoy FJ, Mattson DL. Effect of renal medullary circulation on arterial pressure. J Hypertens Suppl 1992; 10: S187-S193.

10. Mattson DL, Roman RJ, Cowley AW Jr. Role of nitric oxide in renal papillary blood flow and sodium excretion. Hypertension 1992; 19: 766-769.

11. Ruilope LM, Garcia RR, Paya C, Alcazar JM, Miravalles E, Sancho-Rof J, et al. Effects of long-term treatment with indomethacin on renal function. Hypertension 1986; 8: 677684.

12. Strick DM, Fiksen-Olsen MJ, Lockhart JC, Roman RJ, Romero JC. Direct measurement of renal medullary blood flow in the dog. Am J Physiol 1994; 267: R253-R259.

13. Kleinman LI, Radford EP Jr. Ventilation standards for small mammals. J Appl Physiol 1964; 19: 360-362.

14. Bruning JL, Kinz BL. Computational handbook of statistics. Glenview: Scott-Foresman; 1977.

15. Zar JH. Biostatistical analysis. Englewood Cliffs: Prentice Hall; 1974.
16. Donker AJ, Arisz L, Brentjens JR, van der Hem GK, Hollemans $\mathrm{HJ}$. The effect of indomethacin on kidney function and plasma renin activity in man. Nephron 1976; 17: 288-296.

17. Blasingham MC, Nasjletti A. Differential renal effects of cyclooxygenase inhibition in sodium-replete and sodiumdeprived dog. Am J Physiol 1980; 239: F360-F365.

18. Majid DS, Godfrey M, Navar LG. Pressure natriuresis and renal medullary blood flow in dogs. Hypertension 1997; 29: 1051-1057.

19. Clive DM, Stoff JS. Renal syndromes associated with nonsteroidal antiinflammatory drugs. N Engl J Med 1984; 310: 563-572.

20. Schnermann J, Briggs JP. Participation of renal cortical prostaglandins in the regulation of glomerular filtration rate. Kidney Int 1981; 19: 802-815.

21. Fenoy FJ, Ferrer P, Carbonell L, Garcia-Salom M. Role of nitric oxide on papillary blood flow and pressure natriuresis. Hypertension 1995; 25: 408-414.

22. Salom MG, Lahera V, Romero JC. Role of prostaglandins and endothelium-derived relaxing factor on the renal response to acetylcholine. Am J Physiol 1991; 260: F145F149.

23. Roman RJ. Pressure-diuresis in volume-expanded rats. Tubular reabsorption in superficial and deep nephrons. Hypertension 1988; 12: 177-183.

24. Lerman LO, Bentley MD, Fiksen-Olsen MJ, Strick DM, Ritman EL, Romero JC. Pressure dependency of canine intrarenal blood flow within the range of autoregulation. $\mathrm{Am}$ J Physiol 1995; 268: F404-F409.

25. Bergstrom G, Evans RG. Mechanisms underlying the antihypertensive functions of the renal medulla. Acta Physiol Scand 2004; 181: 475-486. 